\title{
The Solar Flux and Sunspot Number; A Long-Trend Analysis
}

\author{
Babu Ram Tiwari* and Mukul Kumar \\ State Key Laboratory of Space Weather, National Space Science Center, \\ Chinese Academy of Sciences, Beijing, China-100190 \\ * Corresponding author email: babu.cas@outlook.com
}

Received: 10 June 2018 / Revised: 30 June 2018 / Accepted: 8 July 2018 / Published: 16 July 2018

\begin{abstract}
A BSTRA C T
We investigate the nature of monthly sunspot numbers and solar flux F10.7 by employing the linear and multiple regression techniques. We observed a brilliant correlation between monthly mean sunspot number and F10.7. We observed that even in deep solar minimum there exist some magnetic activities. We obtained the coefficient of determination $\mathrm{R}^{2}$ to be 0.9533 . We estimated the correlation coefficient for solar flux F10.7 and sunspot number to be 0.97. We extrapolated the F10.7 back to the year 1700 and observed a good correspondence between the modelled F10.7 and sunspot nature. We also found a very good correspondence between the modelled and observed solar flux F10.7.
\end{abstract}

Keywords: Sunspots, Solar Flux, Solar Minima, Solar Maxima

\section{Introduction}

Sunspots, the dark regions with concentrated magnetic field and low temperature, found in the photosphere are known to the human race since the middle of the fourth century, coined by Theophrastus [1]. The Sunspots are the temporary structures evolved on the Sun and their population varies according to the eleven-year cycle of solar minimum and maximum. Their growth, survival and decay have been described by different theories, and mechanisms [2-8]. The Sunspots numbers which have been recorded for several centuries are also used as a proxy for describing the level of solar activity. The data of monthly averaged sunspot numbers are available from back to 1749 . The solar radio emissions at the wavelengths were coined by Southworth in 1945 [9]. The Solar flux F10.7 has been treated as one of the most extensively used parameters for the solar activity. The solar flux F10.7 is an extent of the intensity of solar radio emissions measured on a wavelength of $10.7 \mathrm{~cm}$ or on a frequency of $2800 \mathrm{MHz}$. F10.7 is treated as the level indicator of a solar activity and also serves as the proxy for determining other solar quantities. It's been widely applied in astronomy, climate modelling, geophysics, meteorology, communications, and satellite systems etc. The value of F10.7 which is observed as the flux density and widely referred as the flux, in fact, is the evaluation of total solar flux emitted by all the sources on the solar disk, for a one-hour period on a wavelength of $10.7 \mathrm{~cm}$. Because the solar emissions are very much sensitive to the conditions in the upper chromosphere and lower corona, the wavelength region of $10 \mathrm{~cm}$ is widely accepted as the best one for monitoring the level of solar activity.

There have been a number of investigations to space weather prediction e.g. a multiplicative autoregression model for forecasting monthly values of F10.7 [10], forecasting of the solar activity using neural network algorithms [11]. Zhao \& Han [12] reconstructed the development history of F10.7 and put forward a long-term prediction method of solar $10.7 \mathrm{~cm}$ radio flux. 
The present work is focused on the regression analysis of the monthly mean Sunspot number and solar flux F10.7. Although we are in an era which is dominated by the spaceborne observations still the ground-based solar observations at a wavelength of $10.7 \mathrm{~cm}$ have their own importance. The data of the sunspot number analyzed in this work is obtained from the Sunspot Index Data Center (http://www.sidc.be/silso/). We obtained the data for the flux F10.7 index, from https://www.ngdc.noaa.gov/stp/spaceweather/solar-data/solar-features/solarradio/. We considered the length of sunspot data since 1947 because the earliest available observations of F10.7 are from 1947. We employed linear and multiple regression analysis for our investigation.

\section{Results and Discussion}

Figure (1) reveals the variation of monthly sunspot number to the flux F10.7 cm for the period 19472016. The solar flux unit is defined as $10^{-22} \mathrm{~W} \mathrm{~m}^{-2}$ $\mathrm{Hz}^{-1}$. The monthly mean sunspot number and flux F10.7 are being represented by black and green lines, respectively. From the figure (1), we found the nature of the variation of mean sunspot number and flux F10.7 is alike. The magnitude of F10.7 is found to be low for the solar minimum and high for solar maximum. Figure (1) shows that the values of F10.7 and mean sunspot numbers have been highest during solar cycle 19 (1955-1964) and lowest during solar cycle 24 (2008-2015). However, the mean sunspot numbers and F10.7 are found to be consistent in a range during solar cycle 21 (19761986) and solar cycle 22 (1986-1996). We noticed a lesser number of sunspots during the solar cycle 20 (1964-1976) and 23 (1996-2007). During post half solar cycle 18 (1947-1954), we noticed a peak in sunspot number as well as in the case for the flux indicating that it was a solar maximum and was highly active. The figure (1) also reveals that the F10.7 is not zero anywhere, screening that there is always a magnetic activity even during the deep solar minimum. The large variation around the maxima is owing to the appearance and decay of active regions.

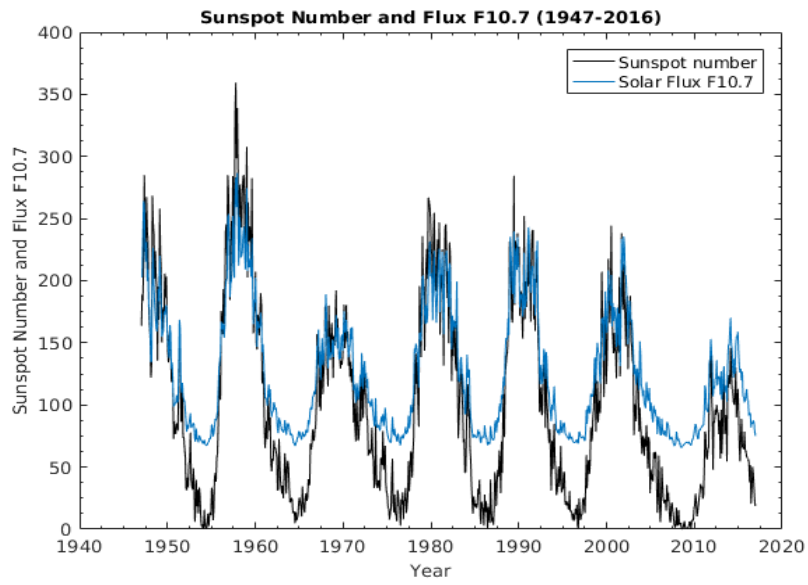

Figure 1: The variation of monthly mean sunspot number and the solar flux F10.7 for the time range 1947-2016. The black and green lines are representing the sunspot number and the F10.7, respectively.

We found that the peak values, duration and shape of solar activities differ for different solar cycles. Figure (2) illustrates the linear regression analysis of monthly averaged sunspot number and F10.7. We have preferred data length since 1947 to reconstruct the variation of sunspot number and solar flux F10.7. We have given efforts in a direction to predict the maximum of F10.7, based on the relationship between the characteristics of the ascending part of the solar cycle and the maximum flux.

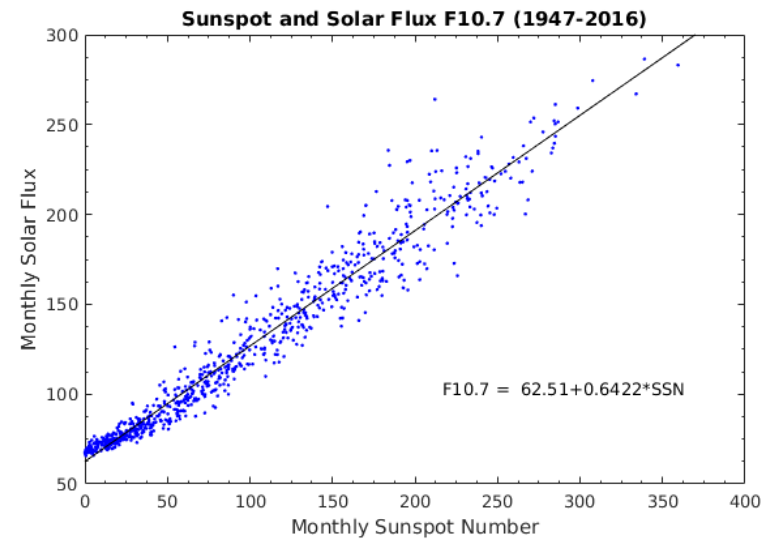

Figure 2: A scatter plot showing the linear regression analysis of mean monthly Sunspot

Numbers and Solar Flux F10.7. The solid line represents the linear fit for the monthly mean Sunspot numbers and flux F 10.7. 
Tiwari et al., Int. Ann. Sci.; Vol. 5, Issue 1, pp: 47-51, 2018

We found an excellent correspondence between sunspot number and F10.7. We obtained the coefficient of determination $\mathrm{R}^{2}$ to be 0.9533 . We also obtained the correlation coefficient for solar flux F10.7 and sunspot numbers to be 0.97. Figure (2) represents the scatter linear plot of the monthly average of sunspot number and F10.7. The solid line in the figure 2 which represent the linear regression analysis is trailing the equation:

$$
\mathrm{F} 10.7=62.51+0.6422 * \mathrm{SSN}
$$

The value of the coefficient of determination $\mathrm{R}^{2}$ is very informative about the correlation of two quantities. Hence, on behalf of the numeric value coefficient of determination $\mathrm{R}^{2}$ which is 0.9533 and for adjusted it is 0.9532 , we conclude that the monthly averaged solar flux and sunspot number are greatly correlated. Our findings of linear regression analysis are in good agreement with Zhao \& Han [12]. Figure (2) also illustrates that the solar flux F10.7 cannot be zero even if the sunspot numbers are zero.

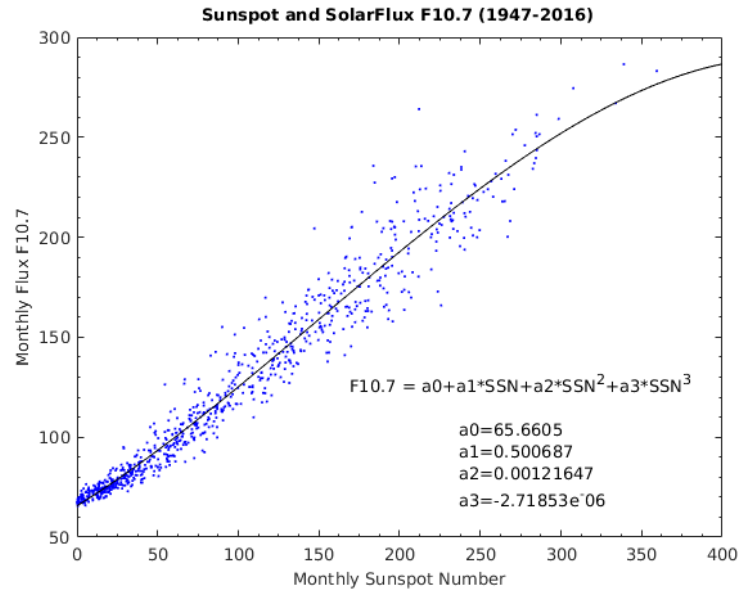

Figure 3: A scatter plot showing the polynomial regression analysis of mean monthly Sunspot

Numbers and Solar Flux F10.7. The solid line represents the polynomial fit for the monthly mean Sunspot numbers and flux F10.7.

Figure (3) characterizes the polynomial regression analysis of order three for monthly averaged sunspot number and F10.7. We have preferred data length same as above (during 1947-2016) to reconstruct the variation of sunspot number and solar flux F10.7. We have also given efforts in a direction to predict the maximum of F10.7, based on the relationship between the characteristics of the ascending part of the solar cycle and the maximum flux. The third-degree polynomial regression analysis represented by the solid line in figure (3) is giving the following equation:

$$
\mathrm{F} 10.7=\mathrm{a} 0+\mathrm{a} 1 * \mathrm{SSN}+\mathrm{a} 2 * \mathrm{SSN}^{\wedge} 2+\mathrm{a} 3 * \mathrm{SSN}^{\wedge} 3
$$

with the coefficients being $\mathrm{a} 0=65.6605$, $\mathrm{a} 1=0.500687, \mathrm{a} 2=0.00121647, \mathrm{a} 3=-2.71853 \times 10^{-6}$ The coefficient of determination for the polynomial regression fit is obtained to be 0.9543 and for adjusted data, it is 0.9542 . Our findings for polynomial regression analysis are in good agreement with Zhao \& Han [12]. Although, the coefficient of determination $\mathrm{R}^{2}$ is nearly same as for linear regression but the polynomial regression provides the better fit for the data. Figure (3) shows that the solar flux F10.7 cannot be zero even if the sunspot numbers are zero, indicative of that there is always some magnetic activity even if the sunspot number is zero in deep solar minimum. We investigated the variation of observed and modelled solar flux F10.7 presented in Figure 4.

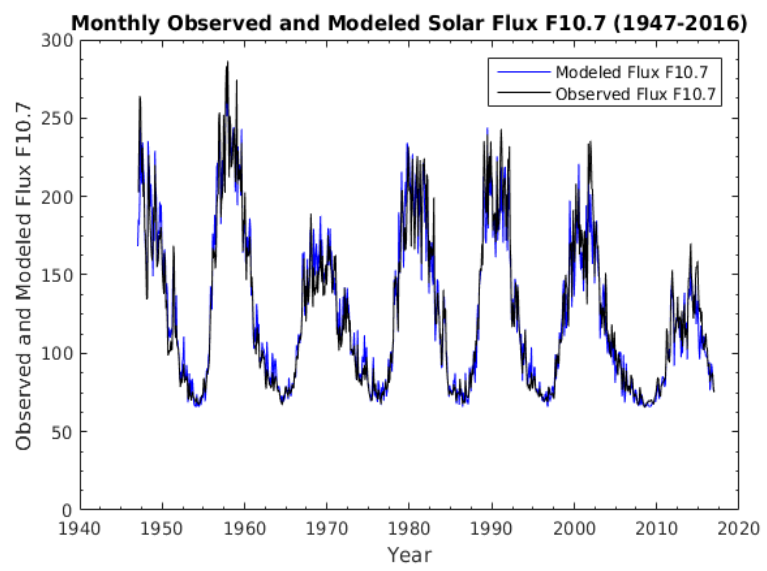

Figure 4: The variation of the observed and modelled solar flux F10.7. The blue and black lines represent the modelled and observed flux F10.7, respectively. Monthly Observed Solar Flux F10.7 and Modeled Solar Flux F10.7.

We modelled the solar flux F10.7 using the third order polynomial equation:

$$
\mathrm{F} 10.7=\mathrm{a} 0+\mathrm{a} 1 * \mathrm{SSN}+\mathrm{a} 2 * \mathrm{SSN}^{\wedge} 2+\mathrm{a} 3 * \mathrm{SSN}^{\wedge} 3
$$


The above equation is solved for the coefficients $\mathrm{a} 0=65.6605, \mathrm{a} 1=0.500687, \mathrm{a} 2=0.00121647, \mathrm{a} 3=-$ $2.71853 \times 10^{-6}$ for the period 1947 to 2016. We found that the modelled and observed solar flux F10.7 shows a brilliant correlation for every solar cycle regardless of solar minimum or maximum. We investigated the month to month error in the modelled and observed time series for the flux F10.7, presented in figure (5). From figure (5), we observe that during the post half solar cycle 18 , solar cycle 19 , solar cycle 20 , solar cycle 22 and solar cycle 23 , the modelled solar flux is found to be consistent with the observed solar flux F10.7. However, for solar cycle 21 and 24 the modelled solar flux F10.7 is found to be a slightly more and lesser than the observed solar flux F10.7, respectively. The minor error found among the modelled and observed solar flux corresponds to a non-zero possibility of occurring a magnetic activity even in deep solar minimum, which is affirmed in the explanation of figure (1).

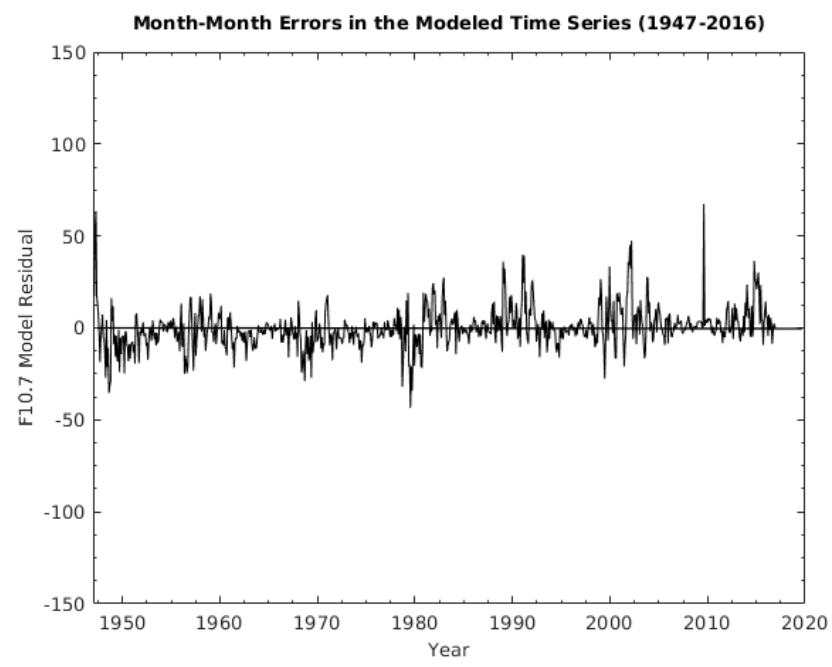

Figure 5: The month-to-month errors in the modelled time series from observed time series for F10.7.

Figure 6 represents the variation of modelled annual mean solar flux F10.7 from 1700 to 2016. Holland \& Vaughan [14] extrapolated the F10.7 data back to 1749 and found a good correlation between the modelled and observed for F10.7. As the observed solar flux is available only since 1947, we extrapolate the solar flux F10.7 using the third order polynomial equation with coefficients a0, a1, a2 and a3. We, likewise, extrapolated F10.7 for a much longer time from 1947 to 2016 and noticed that the observed and modelled F10.7 are superbly correlated. We observed that the values of solar flux F10.7 is in good agreement with the observed solar flux F10.7 during 1947-2016.

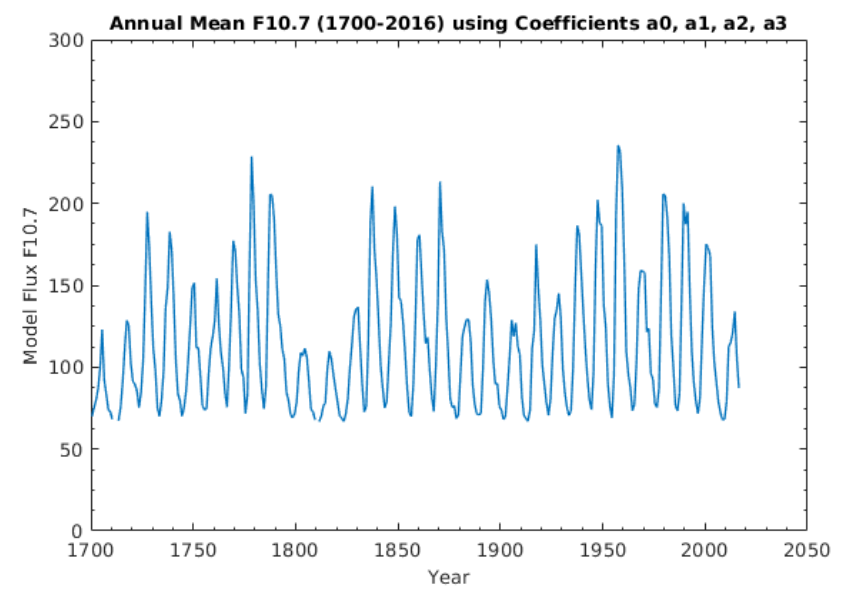

Figure 6: The modelled annual mean of solar flux F10.7

However, at the beginning of $17^{\text {th }}$ century, F10.7 is found to be minimum which corresponds to the Maunder minimum, when the sunspot number was also minimum, hence, we conclude a good correlation between the modelled solar flux and sunspot numbers even during the Maunder solar minimum. At the beginning of the $18^{\text {th }}$ century, F10.7 also found to be minimum and this minimum corresponds to the Dalton minimum when the sunspot number was also minimum, showing an excellent correlation of solar flux and sunspot number during the Dalton minimum period.

\section{Conclusion}

The Sunspot numbers have been widely worn as a proxy for determining the solar activity level because of the availability of sunspot data for the longest duration. Solar flux F10.7 is also a vital quantity in order to define the level of magnetic activities on the Sun. In the present work, we have preferred the monthly mean sunspot numbers and F10.7. We employed the linear as well as multiple regression techniques in our investigation for a longer data set from 1947 to 2016. While comparing our results with previous work, we 
Tiwari et al., Int. Ann. Sci.; Vol. 5, Issue 1, pp: 47-51, 2018

observed that the value of the coefficient of determination, $\mathrm{R}^{2}$ obtained by us $\left(\mathrm{R}^{2}=0.9533\right)$ is vaguely smaller than that of previous result. The difference in the coefficient of determination may correspond to a decrease in magnetic activity during the time period 2005 to 2016. For the case of linear regression, we see that the flux F10.7 is progressively increasing in a linear manner with the sunspot number being in good agreement. We observed an excellent correlation between the modelled and observed F10.7. While cunning the month to month error in the time series of modelled and observed flux F10.7 for the solar cycles 19 to 24, we found that the modelled F10.7 is consistent with all the solar cycles but a minute error in solar 21 and 24. As the solar flux F10.7 is not available previous to 1947 , extrapolated the F10.7 data back to 1749 and found a good correlation between the modelled and observed for F10.7. We, likewise, extrapolated F10.7 for a much longer time from 1947 to 2016 and noticed that the observed and modelled F10.7 are superbly correlated. We modelled the F10.7 back to 1700 and found that the modelled F10.7 shows a very good correlation with the sunspot number including the Maunder minimum and Dalton minimum period.

Finally, as concluding remarks, we would like to point out that the coefficient of determination which is found to be 0.95 yields a very high correlation between the sunspot number and flux F10.7 even on the monthly average basis, hence, for the studies in Solar-terrestrial physics, consideration of both the SSN or F10.7 is equally good.

\section{Acknowledgements}

Authors acknowledge the PIFI postdoctoral fellowship of Chinese Academy of Sciences for financial support and State Key Laboratory of Space Weather for providing the fundamental facilities.

\section{How to Cite this Article:}

B. Tiwari and M. Kumar, "The Solar Flux and Sunspot Number; A Long-Trend Analysis", Int. Ann. Sci., vol. 5, no. 1, pp. 47-51, Jul. 2018. doi: 10.21467/ias.5.1.47-51

\section{References}

[1] Bray R. J., Laughhead R.E., Sunspots, The International Astrophysics Series, London: Chapman \& Hall, 1964.

[2] Cowling T. G., The growth and decay of the sunspot magnetic field, MNRAS, 1946, 106.

[3] Alfven H., On the theory of sunspots, Tellus, 8:2 1956.

[4] Parker E. N., Hydromagnetic Dynamo Models, The Astrophysical Journal, 1955, 122, 293.

[5] Babcock H. W., The topology of the Sun's magnetic field and the 22-year cycle, The Astrophysical Journal, 1961, $133,572$.

[6] Leighton R. B., Transport of magnetic fields on the Sun, The Astrophysical Journal, 1964, 140, 1547.

[7] Zirin H., Fine Structure of Solar Magnetic Field, Solar Physics, 1972, 22, 34-48.

[8] Meyer F., Schmidt H. U., Weiss N. O., Wilson P. R., "The growth and decay of Sunspots", MNRAS, 1974, 169, 3557.

[9] Southworth G. C., Microwave radiation from the Sun, J. Franklin Inst., 1945, 239, 285-29.

[10] Mordvinov A. V., Prediction of monthly mean values of solar activity index F2800 using a multiplicative autoregressive model, CoSka, 1986, 16, 617.

[11] Dmitriev A, Minaeva Yu., Orlov Yu., Riazantseva M., Veselovsky I., Solar Activity Forecasting on 1999-2000 by means of artificial neural networks, Reported on EGS XXIV General Assembly, 1999.

[12] Zhao J., Han Y. B., Historical dataset reconstruction and a prediction method of solar $10.7 \mathrm{~cm}$ radio flux, Chin.J. Astron.Astrophys., 2008, 8

[13] Holland R. L., Vaughan W. W., Lagrangian least-square prediction of solar flux F10.7. 1984, 89, 11-16.

Publish your research article in AIJR journals-

$\checkmark$ Online Submission and Tracking

$\checkmark$ Peer-Reviewed

$\checkmark$ Rapid decision

$\checkmark$ Immediate Publication after acceptance

$\checkmark$ Articles freely available online

$\checkmark \quad$ Retain full copyright of your article.

Submit your article at journals.aijr.in

Publish your books with AIJR publisher-

$\checkmark$ Publish with ISBN and DOI.

$\checkmark$ Publish Thesis/Dissertation as Monograph.

$\checkmark$ Publish Book Monograph.

$\checkmark$ Publish Edited Volume/ Book.

$\checkmark$ Publish Conference Proceedings

$\checkmark \quad$ Retain full copyright of your books.

Submit your manuscript at books.aijr.org 